\title{
The Design and Develop of A Laser Shaft Alignment Instrument Based on The Two Dimensional PSD
}

\author{
Mu Li*, Wang Xinwei \\ Department of Mechanical Engineering, Shenyang Ligong university, LiaoNing Shenyang 110159, China \\ *Corresponding author's Email: muli98@sohu.com
}

\begin{abstract}
A laser shaft alignment based on double LD-PSD is designed in this thesis. The mathematic model is built. The mechanical device which fixes the instrument is designedand the hardware circuit is also designed. The laser shaft alignment has strong anti-jamming capacity. It can compensate the soft ground effect and temperature effect. Therefore, it can carry out alignment measurement under various conditions. The prototype test shows the measuring precision, reliability and anti-jamming ability are higher than the likewise products. It can meet the requirement of coaxiality measuring in shafting.
\end{abstract}

Keywords: PSD; Laser; mechanical device; shaft alignment; Spot

\section{Introduction}

Machine jointed by coupling would generate vibration when running because of shaft misalignment and the vibration affects the using life of machine and damages the parts. As we knowalmost $50 \%$ of the rotating machinery fault is caused by the shaft misalignment. So shaft alignment is an important process in power machinery assembly [1].

The advanced Laser technology is the key technology in measuring and adjusting shaft misalignment. The homemade laser shaft alignment is used as alignment tools and the deviation value is given by manual calculation. It can't take advantage of the laser shaft alignment efficiently. And foreign products are expensive [2]. Thereforelaser shaft alignment product based on double LD-PSD is designed in this thesis. In the actual measurement, the relative position of the two shafts can be calculted by measuring the spot's coordinate of the double LD-PSD on three different angles. The alignment purpose can be realized by adjusting shaft position according to the calculation result. Furthermorethe prototype is designed and used to detecte the actual engineering measurement.

\section{The mathematic model of measurement sys- tem}

There are two deviations in shaft misalignment. One is the parallel deviation. It means two jointed shafts are parallel, but not collinear. Another is the angle deviation. It means two jointed shafts are not parallel and formed angle [3]. When Measuring, usually the two-axis spatial location are decomposed into two mutually perpendicular planes, then the deviation can be calculted using the spatial relationship of the twoaxis, at last adjusting the shaft alignment according to the calculation result.

\subsection{Positional relationship between the drving shaft and driven shaft; the coordinate system es- tablishment}

The double LD-PSD laser shaft alignment and its data acquisiton and processing system are used in the design. 
The optical path system is the basic structure of the laser shaft alignment. The mechanical device is designed and installed as shown in Figure 1. The adjustment quantity of the driven shaft's can be calculated according to the data measured by PSD. It means the mathematical mode is built.

When installing, two pillars are fixed vertically on driving shaft and driven shaft respectively, two pillars's height are roughly equal and the two pillars are adjusted, as shown in Figure 1. Then fixe LD and PSD on each pillar, assuring laser beam is parallel to driving shaft and driven shaft respectively and sensor's photosensitive surface is vertical to laser beam (When producing the instrument, it is ensured that photosensitive surface is parallel to the pillar and the two pillars' height are roughly equal). The positional relationship between driving shaft and driven shaft is shown in Figure 2.

xoy plane is vertical to driving shaft and $x^{\prime} o^{\prime} y^{\prime}$ plane is vertical to driven shaft. The angle between driving shaft and driven shaft is very small, and the distance of two PSD is 1 meter to several meters, so we propose xoy was parallel to $x^{\prime} o^{\prime} y^{\prime}$.

The coordinates built according to driving shaft and driven shaft is shown in Figure 3. Point $A\left(x_{0}, y_{0}, 0\right)$ is

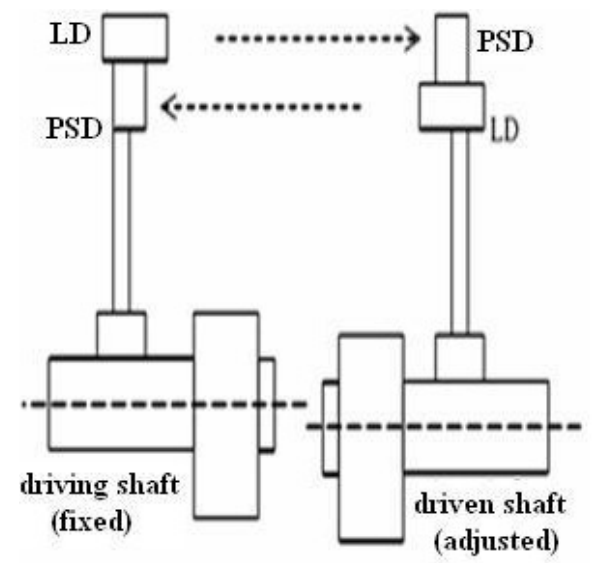

Figure 1 Two-beam LD / PSD diagram

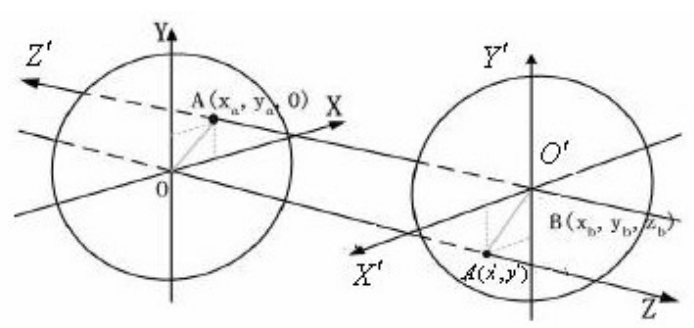

Figure 2 Positional relationship between the driving shaft and driven shaft the intersection of axis and plane, and point $B\left(x_{0}^{\prime}, y_{0}^{\prime}, 0\right)$ is the intersection of $z$ axis and $x^{\prime} o^{\prime} y^{\prime}$ plane.

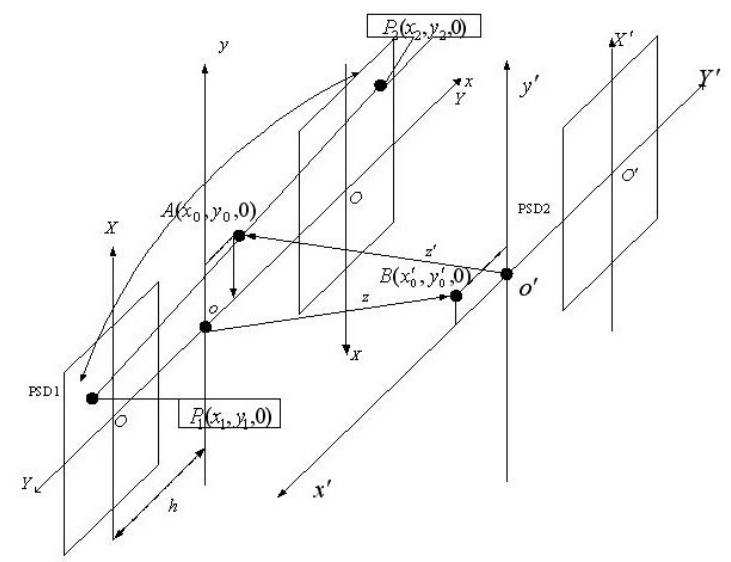

Figure 3 Coordinate established by driving shaft and slave shaft

When measuring, the key process is measuring the coordinates of point $A$ and $B$ precisely, then doing coordinate transformation. Various measuring modes are designed in order to suit different measuring conditions. The coordinate of point $A$ and $B$ can be measured using any three points measurement method.

\subsection{Principle of any three points measurement me- thod}

In Figure 4, point $C$ is the center of PSD fixed on the driving shaft. Point $D$ is the projection of laser beam from the LD fixed on driven shaft to the PSD fixed on driving shaft. When driving and driven shafts are turning a round simultaneously, the track of point $C$ on xoy plane is a circle whose center is point $O$ and radius is $L$; the track of point $D$ is approximate circle whose center is point $A$ and radius is $R$. Point $A$ is the intersection of $z^{\prime}$ and xoy plane. $\sigma$ is the polar coordinate of the spot ouputed by PSD, and its polar radius is $\mathrm{CD}, \alpha$ is the rotation angle. Supposing $A(e, \beta)$ is the polar coordinate of point $A$, then its rectangular is:

$$
A\left(x_{0}, y_{0}\right)=A(e \cdot \cos \beta, e \cdot \sin \beta)
$$

According to The Cosine Theorem, in $\triangle A O D$ :

$$
R^{2}=e^{2}+(L+\sigma)^{2}-2 e(l+\sigma) \cdot \cos (\beta-\alpha)
$$

Then formula (2) is:

$\sigma=\frac{\left(R^{2}-L^{2}\right)-\left(e^{2}-2 e L \cdot \cos (\beta-\alpha)+\sigma^{2}\right)+2 e \delta \cdot \cos (\beta-\alpha)}{2 L}$

In formula (3), when measuring, $R$ is approximately equal to $L$, then formula (3) is:

$$
\sigma=x_{0} \cdot \cos \alpha+y_{0} \cdot \sin \alpha
$$


Similarly, the coordinate of point $B$ is:

$$
\sigma^{\prime}=-x_{0}^{\prime} \cdot \cos \alpha-y_{0}^{\prime} \cdot \sin \alpha
$$

When measuring any three points, the rotation angle is $\alpha_{1}, \alpha_{2}, \alpha_{3}$ respectively, then $\sigma_{1}, \sigma_{2}, \sigma_{3}$ is

$$
\left\{\begin{array}{l}
\sigma_{1}=x_{0} \cdot \cos \alpha_{1}+y_{0} \cdot \sin \alpha_{1} \\
\sigma_{2}=x_{0} \cdot \cos \alpha_{2}+y_{0} \cdot \sin \alpha_{2} \\
\sigma_{3}=x_{0} \cdot \cos \alpha_{3}+y_{0} \cdot \sin \alpha_{3}
\end{array}\right.
$$

According to the least square principle the result can be calculated.

Setting $M=\sum\left[\sigma_{i}-\left(x_{0} \cdot \cos \sigma_{i}+y_{0} \cdot \sin \sigma_{i}\right)\right]$ formula (6) can be solved using formula (7),the result is formula (8).

$$
\left\{\begin{array}{l}
\frac{\partial M\left(x_{0}, y_{0}\right)}{\partial x_{0}}=0 \\
\frac{\partial M\left(x_{0}, y_{0}\right)}{\partial y_{0}}=0
\end{array}\right.
$$

$\left\{\begin{aligned} x_{0} & =\frac{\left.\sum_{(} i=1\right)^{3} \sin ^{2} \alpha_{i} \sum_{i=1}^{3} \sigma_{i} \cos \alpha_{i}-x_{0} \sum_{i=1}^{3} \sin \alpha_{i} \cos \alpha_{i} \sum_{i=1}^{3} \sigma_{i} \sin \alpha_{i}}{\sum_{i=1}^{3} \cos ^{2} \alpha_{i} \sum_{i=1}^{3} \sin ^{2} \alpha_{i}-\left(\sum_{i=1}^{3} \sin \alpha_{i} \cos \alpha_{i}\right)^{2}} \\ y_{0} & =\frac{\left.\sum_{i} i=1\right)^{3} \cos ^{2} \alpha_{i} \sum_{i=1}^{3} \sigma_{i} \sin \alpha_{i}-x_{0} \sum_{i=1}^{3} \sin \alpha_{i} \cos \alpha_{i} \sum_{i=1}^{3} \sigma_{i} \cos \alpha_{i}}{\sum_{i=1}^{3} \cos ^{2} \alpha_{i} \sum_{i=1}^{3} \sin ^{2} \alpha_{i}-\left(\sum_{i=1}^{3} \sin \alpha_{i} \cos \alpha_{i}\right)^{2}}\end{aligned}\right.$

Then $x_{0}$ and $y_{0}$ can be calculated according to formula (9).

$\left\{\begin{aligned} x_{0} & =\frac{\left.\sum_{i} i=1\right)^{3} \sin ^{2} \alpha_{i} \sum_{i=1}^{3} \sigma_{i} \cos \alpha_{i}-x_{0} \sum_{i=1}^{3} \sin \alpha_{i} \cos \alpha_{i} \Sigma_{i=1}^{3} \sigma_{i} \sin \alpha_{i}}{\sum_{i=1}^{3} \cos ^{2} \alpha_{i} \sum_{i=1}^{3} \sin ^{2} \alpha_{i}-\left(\sum_{i=1}^{3} \sin \alpha_{i} \cos \alpha_{i}\right)^{2}} \\ y_{0} & =\frac{\left.\sum_{i} i=1\right)^{3} \cos ^{2} \alpha_{i} \sum_{i=1}^{3} \sigma_{i} \sin \alpha_{i}-x_{0} \sum_{i=1}^{3} \sin \alpha_{i} \cos \alpha_{i} \sum_{i=1}^{3} \sigma_{i} \cos \alpha_{i}}{\sum_{i=1}^{3} \cos ^{2} \alpha_{i} \sum_{i=1}^{3} \sin ^{2} \alpha_{i}-\left(\sum_{i=1}^{3} \sin \alpha_{i} \cos \alpha_{i}\right)^{2}}\end{aligned}\right.$

Similarly, the coordinate of $\left(x_{0}^{\prime}, y_{0}^{\prime}\right)$ is:

$\left\{\begin{aligned} x_{0}^{\prime} & =\frac{\left.\sum_{(} i=1\right)^{3} \sin ^{2} \alpha_{i} \sum_{i=1}^{3} \sigma_{\cos }^{\prime} \cos \alpha_{i}-x_{0} \sum_{i=1}^{3} \sin \alpha_{i} \cos \alpha_{i} \sum_{i=1}^{3} \sigma_{i}^{\prime} \sin \alpha_{i}}{\sum_{i=1}^{3} \cos ^{2} \alpha_{i} \sum_{i=1}^{3} \sin ^{2} \alpha_{i}\left(\sum_{i=1}^{3} \sin \alpha_{i} \cos i_{i}\right)^{2}} \\ y_{0}^{\prime} & =\frac{\left.\sum_{(} i=1\right)^{3} \cos ^{2} \alpha_{i} \sum_{i=1}^{3} \sigma_{i}^{\prime} \sin _{i}-\alpha_{0} \sum_{i=1}^{3} \sin \alpha_{i} \cos \alpha_{i} \sum_{i=1}^{3} \sigma_{i}^{\prime} \cos \alpha_{i}}{\sum_{i=1}^{3} \cos ^{2} \alpha_{i} \sum_{i=1}^{3} \sin ^{2} \alpha_{i}-\left(\sum_{i=1}^{3} \sin \alpha_{i} \cos \alpha_{i}\right)^{2}}\end{aligned}\right.$

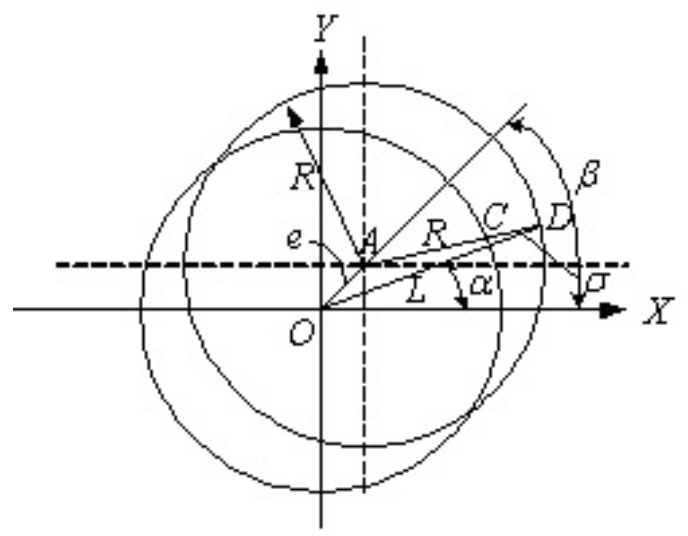

Figure 4 Measurement principle of arbitrary 3 points

\subsection{Deviation and adjustment calculation}

On assuring the positional relationship between the two shafts, the alignment deviation can be calculated according to the coordinate of point $A$ and point $B$. The deviation value can be reduced by adjusting the four anchors of driven shaft. In general, the adjustment method is moving driven shaft in horizontal direction and increasing or decreasing gaskets in vertical direction. The calculation method is given.

The calculation on vertical direction is given as an example in Figure 5.

Point $y_{A}$ and $y_{B}$ are the projections of pioint $A$ and point $B$ on vertical plane, point $k_{y}$ is the projection of the $Z^{\prime}$ shaft on vertical plane, point $E_{x}$ and $F_{y}$ are the projections of the driven shaft motor's front and back anchors. $A$ is the distance between the two PSDs; $b$ is the distance from PSD on the driven shaft to the front anchor, $d$ is the distance from PSD on the driven shaft to the coupling center, $c$ is the distance from the back anchor to the front anchor on driven shaft motor.

Y-coordinates of $E_{y}$ and $F_{y}$ can be calculated by formula (11), according to the geometrical relationship in Figure 5.

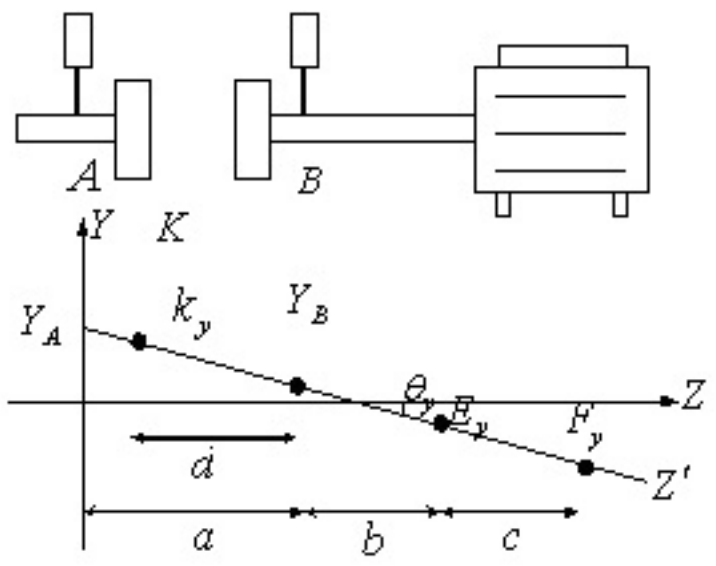

Figure 5 The calculation of slide adjustment and deviation of alignment

$$
\left\{\begin{array}{c}
E_{y}=y_{A}+(a+b)\left(y_{B}-y_{A}\right) / \alpha \\
F_{y}=y_{A}+(a+b+c)\left(y_{B}-y_{A}\right) / \alpha
\end{array}\right.
$$

$E_{y}$ and $F_{y}$ are the adjustment value of the front and back anchors in vertical direction. That is the increasing or decreasing thickness of the gasket. Similarly, $E_{x}$ and $F_{x}$ are the adjustment value of the front and back anchors in horizontal direction

$$
\left\{\begin{array}{c}
E_{x}=x_{A}+(a+b)\left(x_{B}-x_{A}\right) / \alpha \\
F_{x}=x_{A}+(a+b+c)\left(x_{B}-x_{A}\right) / \alpha
\end{array}\right.
$$


$E_{x}$ and $F_{x}$ are the adjustment value of the front and back anchors in horizontal direction. That is the moving distance of the motor.

$\theta_{y}$ is the angle deviation of the two shafts in vertical direction, usually use the Tangent value representation.

$$
\tan \left(\theta_{y}\right)=\frac{Y_{A}-Y_{B}}{\alpha}
$$

Positive and negative signs of the result indicate the deviation direction on vertical plane. Similarly, the angle deviation on parallel plane can be calculated by formula (14).

$$
\tan \left(\theta_{x}\right)=\frac{X_{A}-X_{B}}{\alpha}
$$

\subsection{Principle of temperature compensation}

The position of two center lines change because of high temperature as shown in figure 6 . Therefore the hight difference- $\triangle h t$ appears which causes the misalignment value change. Thus it will fail when the coupling joins the driving shaft and driven shaft. The
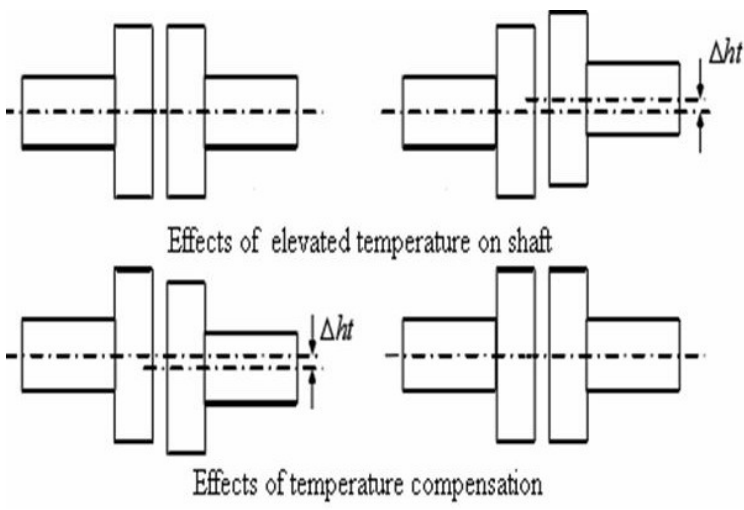

Figure 6 Temperature compensation principle

misalignment deviation caused by the temperature change is not permitted, and it must be suitably amended.

In order to make different seasons on temperature compensation effects become smaller, the temperature compensation is in accordance with the standard of climatic conditions. When the standard of climatic conditions is considered as $20^{\circ} \mathrm{C}$, the temperature compensation can be obtained by formula (15).

$\triangle h t=\left(\triangle t_{2}+t_{0}-20\right) \cdot \mu_{2} \cdot H_{2}-\left(\triangle t_{1}+t_{0}-20\right) \cdot \mu_{1} \cdot H_{1}$

Then the new $E_{y}^{\prime}$ and $F_{y}^{\prime}$ can be calculated by formula (16).

$$
\left\{\begin{array}{c}
E_{y}^{\prime}=Y_{A}+(a+b)\left(Y_{b}-Y_{A}\right) / \alpha+\triangle h_{t} \\
F_{y}^{\prime}=Y_{A}+(a+b+c)\left(Y_{b}-Y_{A}\right) / \alpha+\triangle h_{t}
\end{array}\right.
$$

\section{Laser shaft alignment system based on dou- ble LD-PSD}

\subsection{The composition and measurement principle}

The system composition is shown in Figure 7. The system has five parts: laser source, Optical target, signal processing circuit, data acquisition system and computer.

When measuring, LD and PSD are fixed on the backrest wheel or shaft of the driving shaft and driven shaft respectively using magnetic table block or chain clamp. The laser beam is irradiated by semiconductor laser projects on PSD which fixed on the detected object. A laser spot emerges, when the laser beam projects on the photosensitive surface of PSD. The spot position in the detected object coordinate system can be gained by PSD's signal processing circuit. The coordinates of the detected object in the laser coordinate system can ba obtained according to the spatial relationship between the laser coordinate system and detected object coordinate system. Therefore, the position information of the detected object can be reflected by the PSD's positional information. Then the positional information can be collected to computer by the data acquistion system. The deviation and adjustment quantity can be calculated by programming the software [4].

\subsection{Working principle of the two dimensional PSD}

PSD is a new photoelectric detecting device which is called as the coordinate light battery. It is composed of $\mathrm{P}$ substrate, PIN photodiode and the surface resistance. PSD detects the position of the incident light or particle based on nonuniform semiconductor's "lateral photoelectric effect". So the position of the spot

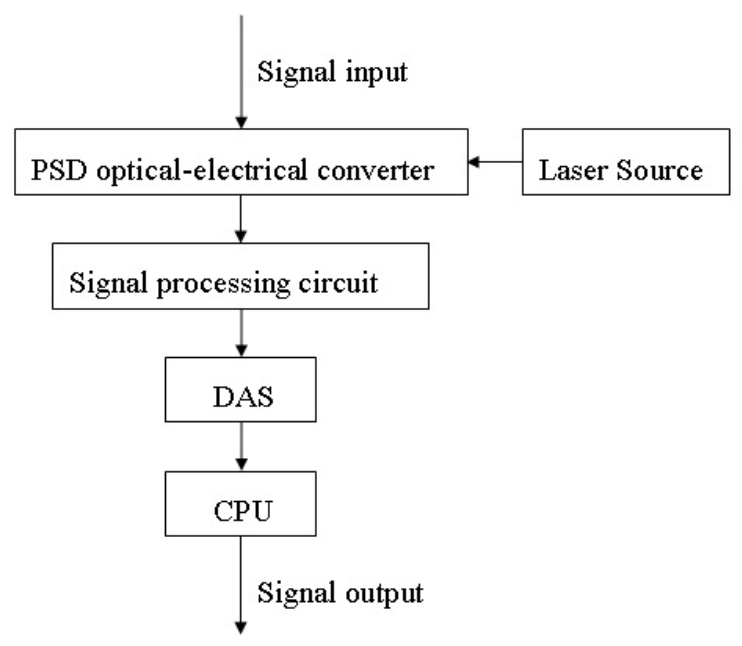

Figure 7 The system block diagram of fixture laser shaft 
energy center which irradiates to the photosensitive area can be measured by PSD's light current and the spot position is transferred to electrical signal. And the positional signal is unrelated to the spot shape. The schematic diagram of two-dimensional PSD is shown in Figure 8 [5].

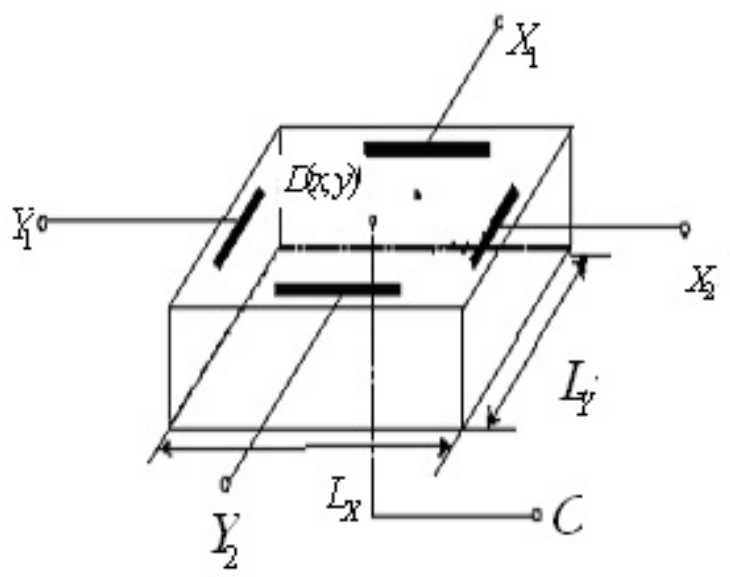

Figure 8 Schematic diagram of two-dimensional PSD

The output current of each Electrode on PSD are $X_{1}, X_{2}, Y_{1}, Y_{2}$. The position of the spot can be calculated by $X_{1}, X_{2}, Y_{1}, Y_{2}$. And the output current can be transformed to voltage by measuring circuit, and the voltage reflects the position of the spot energy center by using the operational amplifier circuit. In Figure 8 , $O$ is the geometric center of PSD, and $O$ is the origin of the PSD coordinate system. $L_{X}$ and $L_{Y}$ are the side lengh of the photosensitive surface in $X$ and $Y$ direction. $(x, y)$ is the coordinates of point $D$. When the laser beam irradiates to point $D$ on the photosensitive surface, a charge generates which is in proportion to light intensity. When the charge crosses the resistance layer, the light current forms and flows to $X_{1}, X_{2}, Y_{1}$, $Y_{2}$. The location coordinate of incoming spot is:

$$
\begin{aligned}
& x=\frac{L_{X}}{2} \frac{X_{2}-X_{1}}{X_{2}+X_{1}} \\
& y=\frac{L_{Y}}{2} \frac{Y_{2}-Y_{1}}{Y_{2}+Y_{1}}
\end{aligned}
$$

Then the polar coordinate of point $D$ can be calculated:

$$
\sigma=\sqrt{x^{2}+y^{2}}
$$

\subsection{Signal Processing Circuit of PSD}

The signal processing circuit of the PSD is shown in Figure 9.
First, the output current would be amplified by the operational amplifiers and be converted to the voltage signal [6]. At the same time, attention must be paid to selecting suitable feedback circuit to ensure that the four circuits of the PSD have the same amplifying performance. Next, adder and subtraction operation would be realized with operational amplifiers. And division operation would be realized with analog divider. Finally, we can obtain a voltage signal that is proportional to the positional signal. And after linking to the computer by high speed A/D converter, we can calculate the shaft misalignment value and the adjustment value.

\subsection{The software design for the measurement sys- tem}

The software is developed with language C. The software diagram is shown in Figure 10. First, acquiring the data from the workpieces joined by the same coupling and calculating. Then, integrating every result. Finally, calculating $E_{x}$ and $F_{x}$ which is the deviation of the shaft misalignment on horizontal plane, calculating $E_{y}$ and $F_{y}$ which is the deviation of the shaft misalignment on the vertical plane. At the same time, calculating $\theta_{x}$ and $\theta_{y}$ which is the angle between the horizontal and vertical plane.

\section{Mechanical device design}

Suitable mechanical device can ensure the signal irradiated by LD can accurately fall in the corresponding PSD and the LD and PSD can be fixed effectively. And it makes the laser alignment instrument remove from the driving shaft and driven shaft conveniently. Therefore, the task is designing a mechnical structure which can fix the LD and PSD firmly on driving shaft and driven shaft respectively. The integral mechanical structure is shown in Figure 11.

There are two designing sections, one is fixture design and the other is laser head structure design .

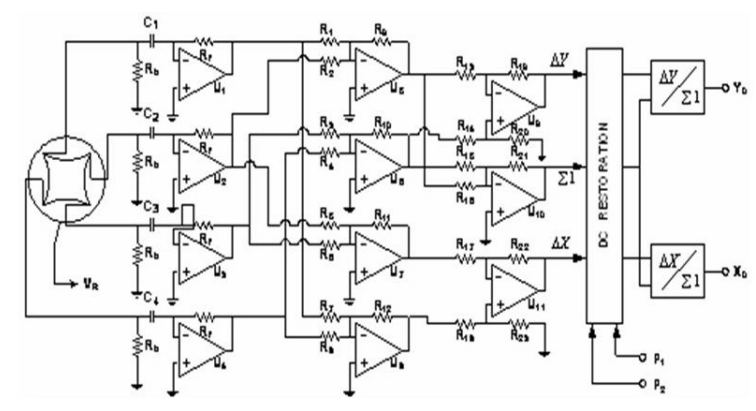

Figure 9 Two-dimensional PSD Signal Processing Circuit 
Fixture design includes type $\mathrm{V}$ block, fixing rod and chain clamping connecting device as shown in Figure 12.

Laser head structure is the core section of laser shaft alignment instrument. It mainly includes LD and PSD. In the laser head structure, laser head can be finetuned, namely it has a trace of adjustment in horizontal direction and vertical direction. Figure 13 is the laser head structure design, Figure 14 is three-view of LD / PSD device.

\section{The experimental results analysis}

In this experiment, we adopt the infrared semiconductor laser as light source, because the peak value of PSD's spectral response is in the infrared region. PSD is the homemade position sensitive photodetec-

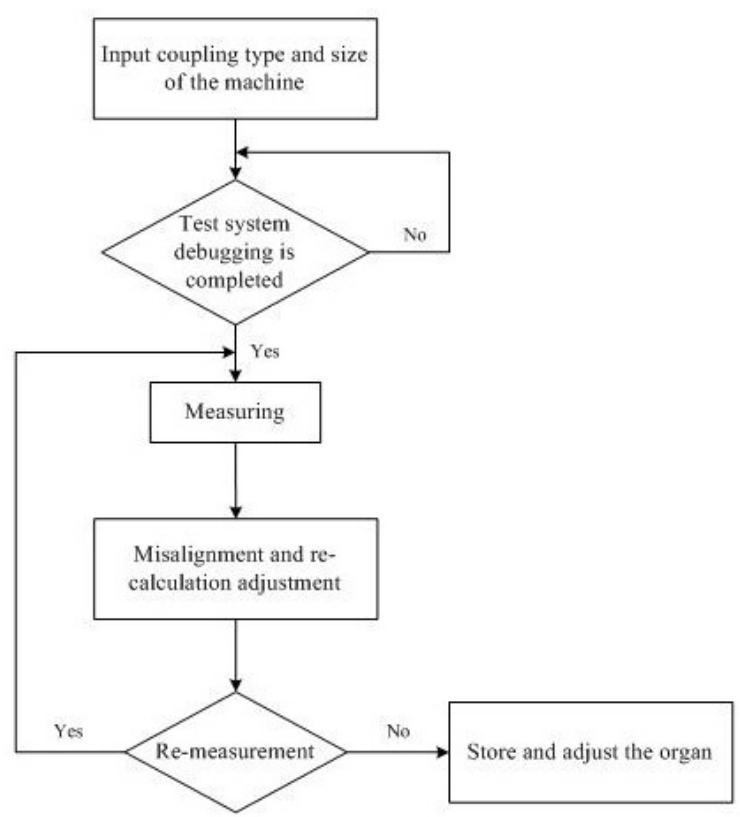

Figure 10 Software system design flow

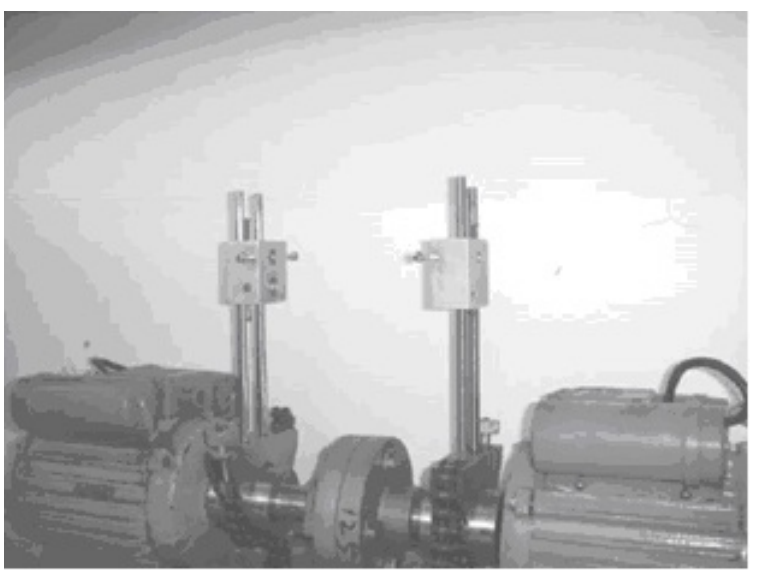

Figure 11 Laser shaft alignment mechanical device tor and the type is GD3284Y. The size of PSD's photosensitive surface is $10 \mathrm{~mm} \times 10 \mathrm{~mm}$, spectral range is $380 \tilde{1} 100 \mathrm{~nm}$, the peak response is $0.6 \mathrm{~A} / \mathrm{W}$, interelectrode resistance is $6 \mathrm{~K} \mathrm{ohm}$, the saturation current value is $0.5 \mathrm{~mA}$, position measurement error is +20 to $+100 \mu \mathrm{m}$ ( intermediate-edge ), the maximum error is $\pm 100 \mu \mathrm{m}$, position resolution is $5 \mathrm{~m}$, the dark current for $20 \mathrm{NA} / \mathrm{Vr}$, the working temperature is -10 to 60 ${ }^{\circ} \mathrm{C}$.

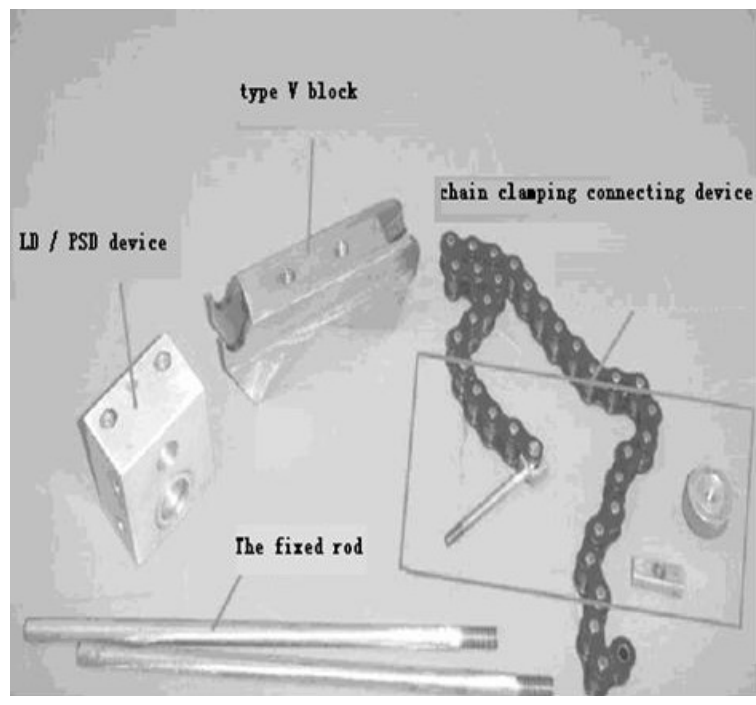

Figure 12 Fixture design

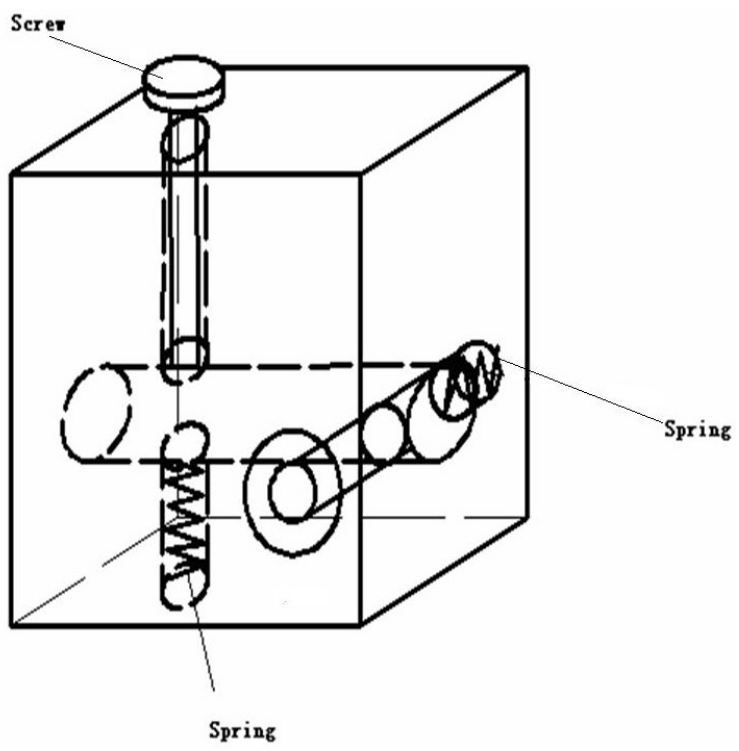

Figure 13 Laser head structure

The diameter of the coupling is $300 \mathrm{~mm}$. The angle deviation between horizontal planes and the angle deviation to vertical plane are both 1 degree. And the coaxial position deviation is $0.30 \mathrm{~mm}$ (deviation in $\mathrm{X}$-axis direction is $0.16 \mathrm{~mm}$, deviation in $\mathrm{Y}$-axis di- 
Table 1 The actual measured Parallel deviation values $(\mathrm{a}=300 \mathrm{~mm})$

\begin{tabular}{|c|c|c|}
\hline \multirow{2}{*}{$\mathbf{D}(\mathbf{m m})$} & \multicolumn{2}{|c|}{ Parallel deviation/mm } \\
\cline { 2 - 3 } & $\begin{array}{c}\text { X direction } \\
\text { deviation }\end{array}$ & $\begin{array}{c}\text { Y direction } \\
\text { deviation }\end{array}$ \\
\hline 300 & 0.058 & 0.059 \\
\hline 400 & 0.062 & 0.065 \\
\hline 500 & 0.067 & 0.064 \\
\hline 600 & 0.069 & 0.068 \\
\hline average value & 0.064 & 0.064 \\
\hline
\end{tabular}

Table 2 The actual measured angle deviation values $(\mathrm{a}=300 \mathrm{~mm})$

\begin{tabular}{|c|c|c|}
\hline \multirow{2}{*}{$\mathbf{D}(\mathbf{m m})$} & \multicolumn{2}{|c|}{ angle deviation/rad } \\
\cline { 2 - 3 } & $\begin{array}{c}\text { horizontal } \\
\text { angle }\end{array}$ & $\begin{array}{c}\text { vertical } \\
\text { angle }\end{array}$ \\
\hline 300 & 0.011 & 0.011 \\
\hline 400 & 0.009 & 0.009 \\
\hline 500 & 0.008 & 0.008 \\
\hline 600 & 0.007 & 0.007 \\
\hline average value & 0.00875 & 0.00825 \\
\hline
\end{tabular}

Table 3 The actual measured deviation values when the distances between the laser and the detector changed

\begin{tabular}{|c|c|c|}
\hline \multirow{2}{*}{ Distance } & \multicolumn{2}{|c|}{ deviation/mm } \\
\cline { 2 - 3 } & $\begin{array}{c}\text { Parallel } \\
\text { deviation }\end{array}$ & $\begin{array}{c}\text { Vertical } \\
\text { deviation }\end{array}$ \\
\hline $\mathrm{a}=200$ & 0.039 & 0.039 \\
\hline $\mathrm{a}=300$ & 0.058 & 0.059 \\
\hline $\mathrm{a}=400$ & 0.077 & 0.078 \\
\hline $\mathrm{a}=500$ & 0.096 & 0.098 \\
\hline $\mathrm{a}=600$ & 0.116 & 0.118 \\
\hline $\mathrm{a}=700$ & 0.135 & 0.138 \\
\hline
\end{tabular}

Table 4 The actual measured angle deviation values when the distances between the laser and the detector changed

\begin{tabular}{|c|c|c|}
\hline \multirow{2}{*}{ Distance } & \multicolumn{2}{|c|}{ angle deviation/rad } \\
\cline { 2 - 3 } & $\begin{array}{c}\text { horizontal } \\
\text { angle }\end{array}$ & $\begin{array}{c}\text { vertical } \\
\text { angle }\end{array}$ \\
\hline $\mathrm{a}=200$ & 0.011 & 0.010 \\
\hline $\mathrm{a}=300$ & 0.011 & 0.011 \\
\hline $\mathrm{a}=400$ & 0.011 & 0.010 \\
\hline $\mathrm{a}=500$ & 0.011 & 0.011 \\
\hline $\mathrm{a}=600$ & 0.011 & 0.012 \\
\hline $\mathrm{a}=700$ & 0.011 & 0.0106 \\
\hline
\end{tabular}

rection is $0.254 \mathrm{~mm}$ ). When the distance between the laser and PSD is $300 \mathrm{~mm}$, the measuring resultsthe shaft alignment deviation in $\mathrm{X}$-axis direction and the shaft alignment deviation in Y-axis direction are shown in Table 1 and Table 2. The parallel deviation and angle deviation in $\mathrm{X}$-axis and $\mathrm{Y}$-axis direction are shown in Table 3 and Table 4, while changing the distance D.
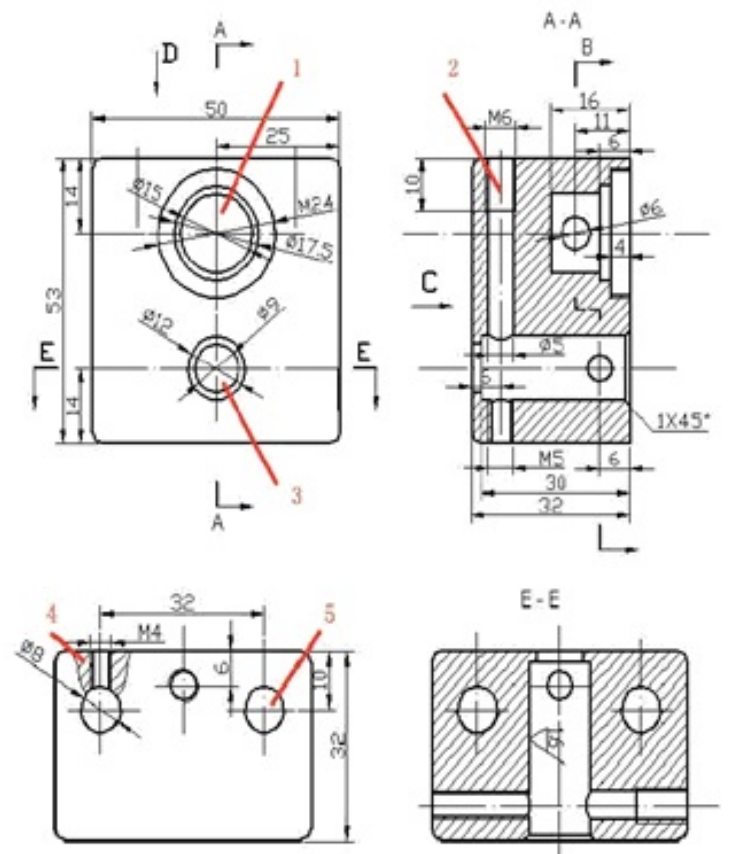

Figure 14 Three-view of LD / PSD

According to GB118796, the shaft misalignment values meet the precision demand of shaft alignmenttolerance Level T14 in geometric tolerance zone.

\section{Conclusion}

This experimental system is simple and easy to use, and can be used by manual or automatic measurement method. The system has automatic collection, transmission and storage data capabilities. It can calculate the data automatically and give the adjustment quantity by software. And the system has good repeatability and strong anti-jamming characteristics. Through several testing, the alignment effect is good, which facilitates adjustment and owns promoting value and many advantages compared with overseas similar products.

\section{References}

[1] Tang JiuYao. "The structure and properties of pillow shaped two-dimensional position sensitive detector", Photonic Sinica, pp.735-738, 2001. 
[2] Jin Guofan, Li Jingzhen. "Laser Measurement Science”, Beijing, Science Press, 1998.

[3] Gao JingWu, Lin Yun and Bai Yun, "Experimental study about two-dimensional PSD in the measurement of position of an object plane", Journal of Eastnorth Electric Power University, pp.46-49, 2005.

[4] Li Zhongke, Zhang Xiaojuan. "Laser fixturlaser shaft mathematical model and computer simulation", $A p$ plication of laser, pp.191-194, 2006.

[5] Cao Guorong, Liu Xiangdong. "Axis adjust the laser detection method", Journal of Agricultural Machinery, pp.92-94, 2001.

[6] Zheng Ergong, Li Hanqiang and Liu Zhiping, "The instrument of an arbitrary corner laser-right based on two-dimensional PSD”, Journal of Wuhan University of Technology, pp.358-361, 2006. 